\title{
Alcohol consumption in relation to carotid subclinical atherosclerosis and its progression: results from a European longitudinal multicentre study
}

\author{
Federica Laguzzi ${ }^{1} \cdot$ Damiano Baldassarre ${ }^{2,3} \cdot$ Fabrizio Veglia $^{3} \cdot$ Rona J. Strawbridge ${ }^{4,5} \cdot$ Steve E. Humphries $^{6}$. \\ Rainer Rauramaa ${ }^{7,8} \cdot$ Andries J. Smit $^{9} \cdot$ Philippe Giral $^{10}$. Angela Silveira ${ }^{5} \cdot$ Elena Tremoli $^{3} \cdot$ Anders Hamsten $^{5}$. \\ Ulf de Faire $^{1,11} \cdot$ Paolo Frumento $^{12} \cdot$ Karin Leander $^{1}$ on behalf of IMPROVE Study group
}

Received: 27 September 2019 / Accepted: 4 March 2020 / Published online: 24 March 2020

(c) The Author(s) 2020

\begin{abstract}
Background/Aim The association between alcohol consumption and subclinical atherosclerosis is still unclear. Using data from a European multicentre study, we assess subclinical atherosclerosis and its 30-month progression by carotid intimamedia thickness (C-IMT) measurements, and correlate this information with self-reported data on alcohol consumption. Methods Between 2002-2004, 1772 men and 1931 women aged 54-79 years with at least three risk factors for cardiovascular disease (CVD) were recruited in Italy, France, Netherlands, Sweden, and Finland. Self-reported alcohol consumption, assessed at baseline, was categorized as follows: none $(0 \mathrm{~g} / \mathrm{d})$, very-low $(0-5 \mathrm{~g} / \mathrm{d})$, low ( $>5$ to $\leq 10 \mathrm{~g} / \mathrm{d})$, moderate $(>10$ to $\leq 20 \mathrm{~g} / \mathrm{d}$ for women, $>10$ to $\leq 30 \mathrm{~g} / \mathrm{d}$ for men) and high ( $>20 \mathrm{~g} / \mathrm{d}$ for women, $>30 \mathrm{~g} / \mathrm{d}$ for men). C-IMT was measured in millimeters at baseline and after 30 months. Measurements consisted of the mean and maximum values of the common carotids (CC), internal carotid artery (ICA), and bifurcations (Bif) and whole carotid tree. We used quantile regression to describe the associations between C-IMT measures and alcohol consumption categories, adjusting for sex, age, physical activity, education, smoking, diet, and latitude.

Results Adjusted differences between median C-IMT values in different levels of alcohol consumption (vs. very-low) showed that moderate alcohol consumption was associated with lower C-IMT ${ }_{\max }[-0.17(95 \% \mathrm{CI}-0.32 ;-0.02)]$, and Bif$\operatorname{IMT}_{\text {mean }}[-0.07(95 \% \mathrm{CI}-0.13 ;-0.01)]$ at baseline and decreasing C-IMT $\mathrm{Imean}[-0.006(95 \% \mathrm{CI}-0.011 ;-0.000)]$, Bif-

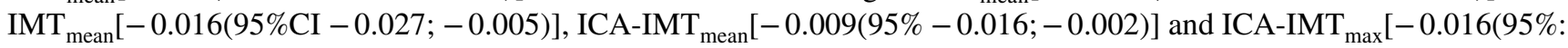
$-0.032 ;-0.000)]$ after 30 months. There was no evidence of departure from linearity in the association between alcohol consumption and C-IMT.

Conclusion In this European population at high risk of CVD, findings show an inverse relation between moderate alcohol consumption and carotid subclinical atherosclerosis and its 30-month progression, independently of several potential confounders.
\end{abstract}

Keywords Alcohol drinking · Atherosclerosis · Carotid intima-media thickness $\cdot$ Progression $\cdot$ Epidemiology

Additional members of the IMPROVE study group are listed in the Supplementary Material.

Electronic supplementary material The online version of this article (https://doi.org/10.1007/s00394-020-02220-5) contains supplementary material, which is available to authorized users.

Federica Laguzzi

federica.laguzzi@ki.se

Extended author information available on the last page of the article

\section{Introduction}

The relation between alcohol consumption and atherosclerosis is still far from established. Atherosclerosis, the main cause of cardiovascular disease (CVD), is a complex chronic low-grade inflammatory disease involving accumulation of lipids and inflammatory markers in the arteries $[1,2]$. Measurements of intima-media thickness in the carotid artery (C-IMT), assessed through simple, non-invasive diagnostic techniques, are considered valid indicators of subclinical atherosclerosis as well as of risk 
of incident CVD [3]. Low-moderate alcohol consumption, corresponding to no more than three standard glasses per day in men and two in women, has previously been shown to exert anti-inflammatory, anti-oxidant, fibrinolytic, and lipid-lowering effects, and to decrease the risk of CVD [4-7]. In contrast, higher alcohol consumption has been associated with increased inflammation, oxidation, and increased risk of CVD $[4,8]$.

Findings from epidemiological studies investigating the association between alcohol consumption and C-IMT have shown inconsistent results: some found a protective effect of moderate alcohol consumptions [9-20], others suggested that alcohol is always a risk factor [21-26], and yet others showed no association [27-35]. Some of the studies have described the relationship between alcohol consumption and atherosclerosis as linear, with either increased [22, 25] or decreased C-IMT [13, 16] associated with a rise in alcohol consumption, whereas others report a J-shaped association, with a decrease of C-IMT with moderate alcohol consumption and an increase of C-IMT with high alcohol consumption $[9,14,15,17]$. Few studies, mainly performed in men [23, 24, 27, 28], often with heavy or binge drinking habits [23, 24, 27], have investigated the relationship between alcohol consumption and progression of atherosclerosis, and results were discrepant $[12,23,24,27,28,36]$.

We aimed to investigate the relationship between alcohol consumption and subclinical atherosclerosis and its 30-month progression in a European multi-centre study including middle-aged men and women at high risk of CVD.

\section{Methods}

\section{Study population}

The Carotid Intima Media Thickness (IMT) and IMT-PROgression as Predictors of Vascular Events in a High-Risk European Population study (IMPROVE) is a European multi-centre study including middle-aged men $(n=1772)$ and women $(n=1931)$ with at least three CVD risk factors. From 2002 to 2004, participants were recruited from seven different centres located in: Italy (two centres: Milan and Perugia), France (Paris), the Netherlands (Groningen), Sweden (Stockholm) and Finland (two centres in Kuopio). The study complies with the Declaration of Helsinki and was approved by the Institutional Review Board of each centre. All patients gave written informed consent. A detailed description of the IMPROVE study is reported elsewhere [37, 38].

The present study was conducted in accordance with the STROBE guidelines [39].

\section{Alcohol consumption assessment}

At baseline, participants were asked to recall their daily consumption of alcoholic beverages in $\mathrm{ml}$ (considering that one glass of wine $\approx 200 \mathrm{ml}$, a pint of beer $\approx 570 \mathrm{ml}$ and a can of beer $\approx 330 \mathrm{ml}$ ) and spirits (one glass of spirit $\approx 25 \mathrm{ml}$ ). From these data, total alcohol consumption per day (g/day) was calculated, considering the different content of alcohol in wine, beer and spirits. We created five categories of alcohol: none $(0 \mathrm{~g} /$ day $)$, very low $[(0,5) \mathrm{g} /$ day $]$, low $[(5,10) \mathrm{g} /$ day], moderate $[(10,20) \mathrm{g} /$ day for women and $(10,30) \mathrm{g} /$ day for men] and high ( $>20 \mathrm{~g} /$ day for women and $>30 \mathrm{~g} / \mathrm{d}$ for men). These categories were created to capture approximately none, half, one, two-three, and above three standard glasses per day, respectively. One standard glass is normally defined as containing 8-12 $\mathrm{g}$ of alcohol and correspond to alcohol content in one bottle of beer $(330 \mathrm{ml})$, one glass of wine $(120 \mathrm{ml})$, or one glass of spirits $(40 \mathrm{ml})$ [40]. Nineteen participants (11 men and 8 women) with missing information on alcohol consumption were excluded from the analyses.

\section{Carotid IMT measurements}

C-IMT, expressed in millimetres $(\mathrm{mm})$, were measured at baseline and after 30 months, by B-mode ultrasonography. For this study, we considered the average of the mean $\left(\mathrm{IMT}_{\text {mean }}\right.$ ) and the maximum (IMT $\mathrm{Imax}_{\text {ma }}$ of the C-IMT measured in the whole carotid arteries and in specific segments i.e. common (CC-IMT mean , CC-IMT $\mathrm{Imax}_{\max }$ ), bifurcation (Bif$\mathrm{IMT}_{\text {mean }}$, Bif-IMT $\mathrm{Imax}_{\text {) and internal (ICA-IMT }}$ mean, ICA$\left.\mathrm{IMT}_{\max }\right)$. The 30-month progression was expressed as mean difference between the 30-month measurement and baseline C-IMT divided for the follow-up time ( $\mathrm{mm} /$ year). Details of the method and its validation are reported elsewhere $[37,38]$. For the progression analysis, 422 participants who dropped out during the follow-up period were excluded.

\section{Possible confounders}

Smoking status was dichotomized in never- and ever-smoker (current or former smoker). Physical activity was categorized into three groups: low (brisk walk for 10 min less than once a week), medium (brisk walk for 10 min at least two-three times/week) and high (brisk walk for 10 min more than three times/week). Education level was categorized into three groups: less than 9 years of school (compulsory school), 9-12 years of school (secondary) and $>12$ years of school (university or college). A score reflecting dietary habits, from 0 to 5 corresponding to level of adherence to a healthy diet, was created as the sum of various dietary items. 
In details, one point was assigned for each of the following dietary habits which were regarded as "healthy": olive oil as main source of type of fat consumed, fish intake more than two times per week, meat intake less than 2 times per week, three or more fruits per day and milk less than $4 \mathrm{dl} /$ day. Based on the recruitment centres, latitude was categorized into six different groups capturing North-South geographical gradient; for descriptive purpose a binary variable (North/South) was created, categorized according to a previous publication [37] Sex and age were also considered as potential confounders.

\section{Statistical methods}

As descriptive statistics, we report the median and the interquartile range (IQR) for continuous variables, and the sample proportions (\%) for categorical variables.

Quantile regression (QR) models at the 50th (p50, median) and 75th percentiles (p75, 3rd quartile) were employed to evaluate the association between alcohol categories and C-IMT measurements at baseline and after 30 months. The rationale for choosing this statistical approach is that it allows the analyst to regress any percentile of the outcome distribution including median and the high percentiles (75th) of the C-IMT [41]. In this population with right skewed C-IMT, the mean values would not provide information on the right tail of the distribution that can also capture abnormal C-IMT indicative of high risk of CVD [42]. Results are delivered as regression coefficients with 95\% confidence intervals (CI). The regression coefficients are interpreted as the 50th and 75th percentile differences in the response variable (a C-IMT measurement) between a specific category of alcohol consumption and the reference category, that corresponds to very low alcohol consumption. Models were adjusted for sex and age (Model 1) plus physical activity, smoking, diet, latitude and education level (Model 2).

To understand the shape of the association between alcohol consumption and the selected percentiles of C-IMT, we also estimated a variation of Model 2 in which we employed restricted cubic splines with four knots at 4, 10, 20 and $30 \mathrm{~g} /$ day to model the effect of alcohol consumption. In this analysis, alcohol consumption was treated as a continuous variable, allowing for a nonlinear effect. These analyses were performed only for those associations that were observed to be significant in the main model. To assess departure from linearity, we tested the nullity of the coefficients associated with the second, third and fourth spline basis.

To verify the robustness of the results, we further adjusted Model 2 for potential mediators of the effect of alcohol consumption on C-IMT. The factors included in the model were: body mass index, high density lipoproteins (HDL), lipid lowering treatments (defined as use of fibrates, statins, omega-3 and resins and used as a proxy for hypercholesterolemia), hypertension (defined as anamnestic or use of antihypertensive treatment or $\mathrm{SBP} \geq 140 \mathrm{mmHg}$ or $\mathrm{DBP} \geq 90 \mathrm{mmHg}$ ) and diabetes (defined as self-reported, or use of anti-diabetic medicine or blood glucose $>7 \mathrm{mmol} / \mathrm{l}$ ).

Based on previous knowledge of sex-specific biological mechanisms in atherosclerosis [43] and that patterns of alcohol consumption and alcohol metabolism vary by sex [44], we performed additional analyses in which men and women were investigated separately. Previous literature on sex-specific associations between alcohol consumption and subclinical atherosclerosis is scarce, in particular in regard to progression.

Sensitivity analyses were performed excluding participants with a CVD event occurring between the time of enrolment and the visit after 30 months.

Missing data were handled by exclusion from each analysis. The total amount of missing data on covariates was less than $4 \%$ for baseline and progression analysis, respectively. A flowchart of the study participants is presented in Figure 1, Supplementary Materials.

Statistical analyses were performed using STATA software (STATA version 12.1, Corp, College Station, TX, USA).

\section{Results}

Table 1 shows the distribution of descriptive characteristics of the IMPROVE participants included in this study $(n=3684)$ and in men and women, separately. The majority of the participants reported no alcohol consumption $(n=1678)$, driven mainly by the large proportion of nonconsumers in women (69\%). Most of the physically active and non-smoking participants, respectively, had very low alcohol consumption whereas the highly educated more often had a moderate or high alcohol consumption.

Hypertension was common among very low consumers of alcohol, and hypertriglyceridemia was common among high consumers. Uric acid was higher among moderate and high consumers, and adiponectin was higher among low consumers. Slightly higher concentrations of total cholesterol and Low Density Lipoprotein (LDL), but not HDL, were also found among moderate and high consumers (vs very low) (Table 1, Supplementary Materials).

Results from analyses of association between alcohol consumption and median C-IMT at baseline are presented in Table 2. When compared to a very low consumption, moderate, high and no alcohol consumption were associ-

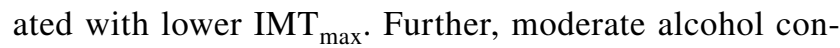
sumption was associated with lower Bif-IMT mean $_{\text {. These }}$ results were independent of confounders included in Model 
2. No clear associations were found for alcohol consumption and $\mathrm{IMT}_{\text {mean }}$, ICA-IMT $\mathrm{Imean}_{\text {. }}$ and ICA-IMT $\mathrm{Imax}_{\text {measured at }}$ baseline.

The associations between alcohol consumption and median C-IMT progression are shown in Table 3. When compared to a very low consumption, any consumption of alcohol (low, moderate and high) was associated with lower $\mathrm{IMT}_{\text {mean }}$ progression. Moreover, moderate and high alcohol consumption were associated with lower Bif-IMT $\mathrm{Imean}_{\text {, }}$ ICA-IMT $_{\text {mean. }}$ and ICA-IMT max $_{\text {max }}$ progression. These results remained significant after the adjustments in Model 2. For the progression, no associations were found for $\mathrm{IMT}_{\max }$ and CC-IMT.

No departure from linearity $(p>0.05)$ was found for the associations between alcohol consumption and median C-IMT at baseline (Fig. 1a) and progression (Fig. 1b).

Analysis of the association between alcohol consumption and the 75th percentile of C-IMT showed that moderate and no alcohol consumption were associated with lower

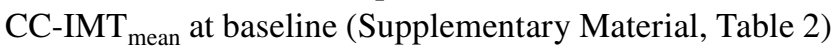
whereas no clear associations were found with C-IMT progression (Supplementary Material, Table 3). An indication of linearity was also shown for the dose-response relationships between alcohol consumption and the 75th percentile of C-IMT (Supplementary Material, Figure 1 A-B).

Results from multivariate analysis with additional adjustment for possible intermediate factors were still significant (data not shown), although the associations between moderate alcohol consumption and Bif-MT $\mathrm{Mean}_{\text {men }}[-0.06(-0.13$; $0.00)]$ and $\mathrm{IMT}_{\text {mean }}[-0.005(-0.011 ; 0.000)]$ progression were slightly attenuated.

Analyses stratified by sex showed associations between alcohol consumption and C-IMT in the same direction as the main analysis (Supplementary Material, Tables 4, 5). Significant associations were found for moderate alcohol consumption and C-IMT $\mathrm{max}_{\max }$ and CC-IMT $\mathrm{Imean}_{\text {, in men, at }}$ baseline, and C-IMT $\mathrm{Imean}_{\text {, ICA-IMT }}$ mean and ICA-IMT $\mathrm{Imax}_{\text {m }}$ in women for the progression. There was a clear relation between moderate alcohol consumption and Bif-IMT mean $_{\text {n }}$ progression both in men and women. However, results were limited by fairly low statistical power.

Regarding the exclusion of participants with CVD events occurring during the period between baseline and the measurements after 30th months $(n=215)$, results were consistent with the main analysis (data not shown).

\section{Discussion}

In this European multi-centre study including participants at high risk of CVD but free of clinical manifestation of CVD at baseline, alcohol consumption was inversely associated, arguably in an approximately linear fashion, with subclinical carotid atherosclerosis and its 30-month progression. These results were independent of sex, age, physical activity, smoking, diet, education and latitude. In particular, compared to very low alcohol consumption, we found that moderate and high alcohol consumption were associated with a lower composite (C-IMT $\mathrm{Tean}_{\text {) }}$ and segment specific (bifurcation and internal carotid) C-IMT progression. At baseline, moderate alcohol consumption was associated with a lower composite $\left(\mathrm{C}-\mathrm{IMT}_{\max }\right)$ and segment specific C-IMT (bifurcations). Lower C-IMT at baseline (C-IMT $\left.{ }_{\max }\right)$ and progression (internal carotids) were also found for the abstainers.

Our findings of moderate alcohol consumption in relation to decreased C-IMT measured at baseline confirm the results of some earlier studies $[9,10,13-17,19,21,36]$ but not all $[11,25,30,32-34]$. Among the few studies $[12,36]$ that have investigated the association between alcohol consumption and progression of atherosclerosis including both men and women, our study is one of the largest. Our findings of lower C-IMT progression in relation to moderate and high alcohol consumption, as compared to very low consumption, agree to some extent with those reported from an Italian study $(n=780)$ [12] but disagree with those of an American study $(n=788)$ [36]. The Italian study observed protective associations also for light-moderate alcohol consumption ( $50 \mathrm{~g} /$ day), compared to abstainers, in their case in relation to atherosclerotic plaque. Compared to our study, participants were healthier and the follow-up was longer (5 years) [12]. The American study was performed in individuals affected by HIV which may hamper comparability between studies due to presence of different confounding factors in the study base [36].

In contrast to previous studies that have found a linear increase [22, 25] or $\mathrm{J}$-shaped curve for the association between alcohol consumption and C-IMT [9, 14, 15, 17], our findings support a linear decrease of C-IMT (both at baseline and after 30-month follow-up) in relation to increasing alcohol consumption. A linear decrease of IMT was previously reported in two other large epidemiological studies $(n>4000)$ including Korean men and women $[13,16]$. The earlier investigations with opposite findings to our study were performed in Americans [15], Chinese [17], Finnish [25], Germans [14, 22] and Italians [9]. Apart from the study population origins being different, the intake of alcohol in our study was generally lower (median $4 \mathrm{~g} / \mathrm{d}$ IQR: $0-16$ ). In our study, only $4 \%$ of all the participants consumed more than $50 \mathrm{~g} / \mathrm{d}$ (corresponding to more than 3 drinks per day), possibly explaining the discrepant findings. In addition, compared with the compared studies, our population was at higher risk of CVD; in subjects with metabolic disturbances and chronic low-grade inflammation, alcohol consumption may attenuate the effect of the risk factors for atherosclerosis [22]. Moreover, a large proportion of our study participants at high risk of CVD were under pharmacological 
Table 1 Baseline characteristics by different levels of alcohol consumption of IMPROVE study participants

\begin{tabular}{|c|c|c|c|c|c|}
\hline Characteristic & Abstainers $(0 \mathrm{~g} / \mathrm{d})$ & Very Low $(>0-5 \mathrm{~g} / \mathrm{d})$ & Low $(>5-10 \mathrm{~g} / \mathrm{d})$ & Moderate $(>10-30 \mathrm{~g} / \mathrm{d})^{\mathrm{a}}$ & $\operatorname{High}(>30 \mathrm{~g} / \mathrm{d})^{\mathrm{b}}$ \\
\hline \multicolumn{6}{|l|}{$n$} \\
\hline All & 1678 & 225 & 375 & 738 & 668 \\
\hline Men & 515 & 119 & 179 & 468 & 480 \\
\hline Women & 1163 & 106 & 196 & 270 & 188 \\
\hline \multicolumn{6}{|c|}{ Total alcohol (g/d) } \\
\hline All & $0(0 ; 0)$ & $4(1.9 ; 4)$ & $8(8 ; 8)$ & $16(16 ; 16.8)$ & $36(32 ; 48)$ \\
\hline Men & $0(0 ; 0)$ & $3.6(2 ; 4)$ & $8(8 ; 8)$ & $16(16 ; 21.6)$ & $40(32 ; 56)$ \\
\hline Women & $0(0 ; 0)$ & $4(1.8 ; 4)$ & $8(8 ; 8)$ & $16(16 ; 16)$ & $32(32 ; 33)$ \\
\hline \multicolumn{6}{|l|}{ Age (y) } \\
\hline All & $64.4(59.6 ; 67.3)$ & $65.3(60.5 ; 67.4)$ & $65.6(60 ; 67.2)$ & $65.2(59.5 ; 67.2)$ & $63.4(59.1 ; 67)$ \\
\hline Men & $64.6(59.5 ; 67.1)$ & $65.3(59.9 ; 67.3)$ & $64.9(59.3 ; 67.3)$ & $65.7(59.3 ; 67.2)$ & $63.2(59.1 ; 66.9)$ \\
\hline Women & $64.2(59.7 ; 67.5)$ & $65.2(61.4 ; 67.8)$ & $65.9(60.1 ; 67.1)$ & $65(59.8 ; 67.2)$ & $63.6(59.4 ; 67.4)$ \\
\hline \multicolumn{6}{|c|}{ Physical activity (\%) ${ }^{\mathrm{m} 3}$} \\
\hline \multicolumn{6}{|l|}{ All } \\
\hline Low & 22.3 & 8.4 & 16.3 & 18.0 & 21.6 \\
\hline Medium & 43.7 & 42.2 & 42.8 & 42.9 & 49.4 \\
\hline High & 34.0 & 49.3 & 40.9 & 39.0 & 29.0 \\
\hline \multicolumn{6}{|l|}{ Men } \\
\hline Low & 16.0 & 7.6 & 14.0 & 13.7 & 20.2 \\
\hline Medium & 42.6 & 41.2 & 41.6 & 40.2 & 49.4 \\
\hline High & 41.4 & 51.3 & 44.4 & 46.1 & 30.4 \\
\hline \multicolumn{6}{|l|}{ Women } \\
\hline Low & 25.0 & 9.0 & 18.0 & 25.6 & 25.0 \\
\hline Medium & 44.2 & 43.0 & 44.0 & 47.8 & 49.5 \\
\hline High & 30.7 & 47.0 & 38.0 & 26.7 & 25.5 \\
\hline \multicolumn{6}{|l|}{ Ever smoker (\%) } \\
\hline All & 13.3 & 10.2 & 14.7 & 15.7 & 19.2 \\
\hline Men & 14.9 & 10.1 & 19.0 & 16.7 & 18.9 \\
\hline Women & 12.6 & 10.4 & 10.7 & 14.1 & 19.7 \\
\hline \multicolumn{6}{|c|}{ Education (\%) ${ }^{\mathrm{m} 34}$} \\
\hline \multicolumn{6}{|l|}{ All } \\
\hline$\leq 9$ years & 51.6 & 44.6 & 46.4 & 39.6 & 38.5 \\
\hline $9-12$ years & 25.3 & 25.2 & 23.2 & 26.3 & 27.0 \\
\hline$>12$ years & 23.0 & 30.2 & 30.5 & 34.1 & 34.4 \\
\hline \multicolumn{6}{|l|}{ Men } \\
\hline$\leq 9$ years & 44.9 & 50.0 & 44.6 & 37.1 & 37.2 \\
\hline $9-12$ years & 25.2 & 23.7 & 21.5 & 24.3 & 27.3 \\
\hline$>12$ years & 30.0 & 26.3 & 33.9 & 38.6 & 35.5 \\
\hline \multicolumn{6}{|l|}{ Women } \\
\hline$\leq 9$ years & 54.5 & 38.5 & 47.9 & 43.8 & 41.9 \\
\hline $9-12$ years & 25.5 & 26.9 & 24.7 & 29.7 & 26.3 \\
\hline$>12$ years & 20.0 & 34.6 & 27.3 & 26.4 & 31.7 \\
\hline \multicolumn{6}{|l|}{ Dietscore $^{\mathrm{cm} 14}$} \\
\hline All & $2(1 ; 3)$ & $1(0 ; 2)$ & $2(1 ; 3)$ & $2(1 ; 3)$ & $2(1 ; 3)$ \\
\hline Men & $1(1 ; 2)$ & $1(1 ; 2)$ & $1(1 ; 2)$ & $1(1 ; 2)$ & $2(1 ; 3)$ \\
\hline Women & $2(1 ; 3)$ & $1(0 ; 2)$ & $2(1 ; 3)$ & $2(1 ; 3)$ & $2(2 ; 3)$ \\
\hline \multicolumn{6}{|c|}{ Geographical gradient $(\%)^{\mathrm{d}}$} \\
\hline \multicolumn{6}{|l|}{ All } \\
\hline North & 57.0 & 93.0 & 62.0 & 59.0 & 40.5 \\
\hline South & 43.0 & 7.0 & 38.0 & 41.0 & 59.0 \\
\hline
\end{tabular}


Table 1 (continued)

\begin{tabular}{|c|c|c|c|c|c|}
\hline Characteristic & Abstainers $(0 \mathrm{~g} / \mathrm{d})$ & Very Low $(>0-5 \mathrm{~g} / \mathrm{d})$ & Low $(>5-10 \mathrm{~g} / \mathrm{d})$ & Moderate $(>10-30 \mathrm{~g} / \mathrm{d})^{\mathrm{a}}$ & $\operatorname{High}(>30 \mathrm{~g} / \mathrm{d})^{\mathrm{b}}$ \\
\hline \multicolumn{6}{|l|}{ Men } \\
\hline North & 66.0 & 97.0 & 76.0 & 70.0 & 44.0 \\
\hline South & 34.0 & 3.0 & 24.0 & 30.0 & 56.0 \\
\hline \multicolumn{6}{|l|}{ Women } \\
\hline North & 53.0 & 88.7 & 48.9 & 40.0 & 32.0 \\
\hline South & 47.0 & 11.0 & 49.0 & 60.0 & 67.5 \\
\hline \multicolumn{6}{|c|}{ Lipid-lowering drugs $(\%)^{\text {e m63 }}$} \\
\hline All & 49.0 & 43.0 & 50.0 & 45.0 & 54.5 \\
\hline Men & 46.0 & 43.0 & 43.0 & 44.0 & 55.0 \\
\hline Women & 51.0 & 44.0 & 56.0 & 47.5 & 54.3 \\
\hline
\end{tabular}

Results are presented for all the participants $(n=3684)$, in men $(n=1761)$ and in women $(n=1923)$, respectively. Median and interquartile range (in brackets) for continuous variables where not specified; proportions for binary and categorical variables (\%)

$m$ missing values

${ }^{\mathrm{a}}$ For women cut-off $>10-<20 \mathrm{~g} /$ day

${ }^{\mathrm{b}}$ For women cut-off $>20 \mathrm{~g} /$ day

${ }^{c}$ Dietscore continuous variable created as described in the Method section

${ }^{\mathrm{d}}$ North includes Finland (2 centers in Kuopio), Sweden (Stockholm), The Netherlands (Groningen); South: France (Paris), Italy (1 center in Milan, 1 center in Perugia)

${ }^{\mathrm{e}}$ Hypolipidemic treatment including statins, fibrate, resins

poly-therapy (including drugs with pleiotropic effect such as statins) that may also have altered the effects of alcohol on C-IMT, regardless of the amount of alcohol consumed [45]. Nonetheless, when we controlled for lipid lowering treatment including statins, the associations were only slightly attenuated.

The biological mechanisms behind a potentially causal protective effect exerted by moderate alcohol consumption on subclinical atherosclerosis and CVD are not completely understood. Epidemiological and experimental studies have suggested that low-moderate (up to three standard drinks) doses of alcohol consumption may have a beneficial effect on the cascade of factors (e.g. lipoprotein, coagulation, adiponectin, inflammatory chemokines, vascular endothelial growth factors) that lead to the formation of atherosclerotic plaques $[4,5,46,47]$. On the other hand, high alcohol consumption may drive the formation of higher amount of the toxic metabolite acetaldehyde. In turn, this may lead to the formation of biological markers involved in the development of the atherosclerotic process [4].

Our results of differential associations referred to different carotid segments, observed at baseline and progression and in men and women separately, are relatively complex to interpret. Carotid subclinical atherosclerosis measured in different segments has been suggested to have different clinical significance; CC-IMT may reflect hyperplasia or hypertrophy of smooth cells strongly related to age, whereas Bif-IMT and ICA-IMT may indicate a pathological response to low shear stress leading to the development of abnormal carotid atherosclerosis [48]. Also, CVD risk factors and atherosclerotic progression are more strongly associated with Bif-IMT and ICA-IMT than with CC-IMT [48]. We found a consistent protective association between alcohol and BifIMT (both at baseline and at progression), and a non-consistent association with CC-IMT and ICA-IMT. Both findings appear reasonable in the light of previous observations.

\section{Strengths and limitations}

A strength of this study is that it is based on a unique cohort with a large sample size, including both men and women, and with availability of data from several C-IMT segments, allowing to capture different physiological and clinical profiles. Importantly, C-IMT measurements were validated and followed a common protocol for all centres. We cannot exclude, however, that some of the results could be false positives. However, the proportion of significant findings (36\% at baseline, and 23\% at progression) was much larger than the $5 \%$ false positive that could be expected by chance under the null hypothesis.

Our results showed robustness against additional adjustment for CVD risk factors. Obviously, we cannot exclude that other possible unmeasured and/or unknown factors that we have not controlled for may explain the observed associations.

Another strength of our study is that we used as reference category the very low consumers; low alcohol consumption has lately been considered a more appropriate group of 
Table 2 Median differences (95\% CI) of IMT measured at baseline in relation to alcohol consumption categories

\begin{tabular}{|c|c|c|c|c|c|c|}
\hline \multirow[t]{2}{*}{ IMT Baseline } & & Abstainers $(0 \mathrm{~g} / \mathrm{d})$ & Very low $(>0-5 \mathrm{~g} / \mathrm{d})$ & Low $(>5-10 \mathrm{~g} / \mathrm{d})$ & Moderate $(>10-30 \mathrm{~g} / \mathrm{d})^{\mathrm{a}}$ & $\operatorname{High}(>30 \mathrm{~g} / \mathrm{d})^{\mathrm{b}}$ \\
\hline & Models & $\beta_{1}(95 \% \mathrm{CI})$ & Reference & $\beta_{1}(95 \% \mathrm{CI})$ & $\beta_{1}(95 \% \mathrm{CI})$ & $\beta_{1}(95 \% \mathrm{CI})$ \\
\hline \multicolumn{7}{|l|}{$\mathrm{IMT}_{\text {mean }} \mathrm{m}^{2}$} \\
\hline \multirow[t]{2}{*}{$\mathrm{p} 50$} & Model 1 & $-0.06(-0.09 ;-0.03)$ & - & $-0.05(-0.09 ;-0.01)$ & $-0.07(-0.1 ;-0.04)$ & $-0.09(-0.12 ;-0.06)$ \\
\hline & Model 2 & $-0.02(-0.05 ; 0.01)$ & - & $0.00(-0.04 ; 0.03)$ & $-0.02(-0.05 ; 0.01)$ & $-0.02(-0.05 ; 0.01)$ \\
\hline \multicolumn{7}{|l|}{$\mathrm{IMT}_{\max }{ }^{\mathrm{m} 2}$} \\
\hline \multirow[t]{2}{*}{ p50 } & Model 1 & $-0.32(-0.48 ;-0.16)$ & - & $-0.25(-0.43 ;-0.06)$ & $-0.33(-0.50 ;-0.16)$ & $-0.39(-0.56 ;-0.22)$ \\
\hline & Model 2 & $-0.18(-0.32 ;-0.04)$ & - & $-0.11(-0.27 ; 0.06)$ & $-0.17(-0.32 ;-0.02)$ & $-0.16(-0.32 ;-0.01)$ \\
\hline \multicolumn{7}{|l|}{$\mathrm{CC}-\mathrm{IMT}_{\text {mean }}{ }^{\mathrm{m} 4}$} \\
\hline \multirow[t]{2}{*}{$\mathrm{p} 50$} & Model 1 & $-0.02(-0.04 ; 0.00)$ & - & $-0.02(-0.04 ; 0.00)$ & $-0.03(-0.05 ;-0.01)$ & $-0.03(-0.05 ;-0.01)$ \\
\hline & Model 2 & $0.00(-0.02 ; 0.02)$ & - & $0.00(-0.02 ; 0.02)$ & $-0.01(-0.03 ; 0.01)$ & $0.00(-0.02 ; 0.02)$ \\
\hline \multicolumn{7}{|l|}{ Bif-IMT $\mathrm{Imean}^{\mathrm{m} 2}$} \\
\hline \multirow[t]{2}{*}{ p50 } & Model 1 & $-0.12(-0.18 ;-0.07)$ & - & $-0.08(-0.15 ;-0.01)$ & $-0.16(-0.22 ;-0.10)$ & $-0.15(-0.21 ;-0.09)$ \\
\hline & Model 2 & $-0.04(-0.10 ; 0.02)$ & - & $0.00(-0.07 ; 0.06)$ & $-0.07(-0.13 ;-0.01)$ & $-0.05(-0.11 ; 0.02)$ \\
\hline \multicolumn{7}{|l|}{ ICA IMT $_{\text {mean }}{ }^{\mathrm{m} 34}$} \\
\hline \multirow[t]{2}{*}{$\mathrm{p} 50$} & Model 1 & $-0.05(-0.09 ;-0.01)$ & - & $-0.05(-0.10 ; 0.00)$ & $-0.06(-0.11 ;-0.02)$ & $-0.09(-0.14 ;-0.05)$ \\
\hline & Model 2 & $-0.03(-0.07 ; 0.02)$ & - & $-0.03(-0.08 ; 0.02)$ & $-0.03(-0.07 ; 0.02)$ & $-0.05(-0.09 ; 0.00)$ \\
\hline \multicolumn{7}{|l|}{ CC-IMT ${ }_{\max }{ }^{\mathrm{m} 4}$} \\
\hline \multirow[t]{2}{*}{$\mathrm{p} 50$} & Model 1 & $-0.04(-0.08 ; 0.01)$ & - & $-0.02(-0.07 ; 0.02)$ & $-0.04(-0.08 ; 0.00)$ & $-0.05(-0.10 ;-0.01)$ \\
\hline & Model 2 & $0.00(-0.04 ; 0.04)$ & - & $0.01(-0.04 ; 0.05)$ & $-0.01(-0.05 ; 0.03)$ & $0.00(-0.04 ; 0.04)$ \\
\hline \multicolumn{7}{|l|}{ Bif-IMT $_{\max }{ }^{\mathrm{m} 21}$} \\
\hline \multirow[t]{2}{*}{$\mathrm{p} 50$} & Model 1 & $-0.20(-0.33 ;-0.08)$ & - & $-0.12(-0.27 ; 0.03)$ & $-0.25(-0.39 ;-0.12)$ & $-0.29(-0.43 ;-0.16)$ \\
\hline & Model 2 & $-0.04(-0.16 ; 0.08)$ & - & $-0.01(-0.15 ; 0.14)$ & $-0.06(-0.20 ; 0.07)$ & $-0.10(-0.23 ; 0.04)$ \\
\hline \multicolumn{7}{|l|}{ ICA IMT $_{\max }{ }^{\mathrm{m} 34}$} \\
\hline \multirow[t]{2}{*}{$\mathrm{p} 50$} & Model 1 & $-0.12(-0.22 ;-0.01)$ & - & $-0.11(-0.23 ; 0.01)$ & $-0.17(-0.28 ;-0.06)$ & $-0.17(-0.28 ;-0.06)$ \\
\hline & Model 2 & $0.00(-0.10 ; 0.10)$ & - & $0.00(-0.12 ; 0.12)$ & $-0.02(-0.13 ; 0.09)$ & $-0.01(-0.13 ; 0.10)$ \\
\hline
\end{tabular}

Results for all participants of the IMPROVE study $(n=3684)$. Number of observations for each analysis: $\mathrm{IMT}_{\text {mean and }} \mathrm{IMT}_{\text {max }}$ : Model $1, n=3682$;

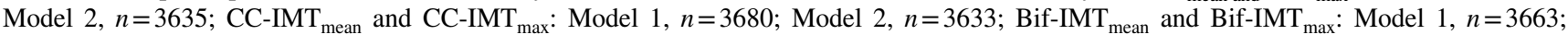
Model 2, $n=3616$; ICA-IMT mean $_{\text {and ICA-IMT }}$ max $:$ Model 1, $n=3650$; Model 2, $n=3603$

Model 1 Adjustments for sex and age; Model 2 Model 1 plus physical activity, education, smoking, latitude (categorical) and diet (continuous); $m$ missing values

${ }^{a}$ For women cut-off $>10$ to $<20$ g/day

${ }^{\mathrm{b}}$ For women cut-off $>20 \mathrm{~g} /$ day

comparison than abstainers $[49,50]$. It is possible that the group of abstainers includes a number of former drinkers who quit due to the presence of comorbidity or metabolic disorder. Such situation would contribute to explain the finding of a lower C-IMT at baseline and at progression for abstainer in comparison to low consumers.

Our study has also some limitations. Alcohol consumption was self-reported and we had no possibility to validate the reported intake of alcohol. Misclassifications may have led to non-differential misclassification of exposure, diluting the estimated effects. Moreover, we do not have repeated measures of alcohol consumption, so we were not able to detect possible changes over the 30-month follow-up.

Although the study is representative of the European population with classical CVD risk factors, the inclusion of different European countries with different drinking patterns may have introduced heterogeneity in the results. Nordic countries are for example known to have a more binge drinking pattern than the Southern European countries. We adjusted for latitude but we were not able to stratify by countries due to lack of statistical power. However, when we stratified by north and south geographical location of centres, results were similar (data not shown).

We cannot rule out the presence of bias due to non-participation at follow-up. However, the mean alcohol consumption was similar in the missing group (mean $12.0 \mathrm{~g} / \mathrm{day}$ sd. $18 \mathrm{~g} /$ day) as compared to the participant group (mean $12.3 \mathrm{~g} /$ day sd. $18 \mathrm{~g} /$ day) making selection bias less likely to affect the internal validity of our study.

Finally, the follow-up for progression of atherosclerosis was fairly short (30 months). However, in an experimental study in mice, a clear decrease of atherosclerotic plaque was already observed after 2 weeks, for daily moderate drinking [51]. 


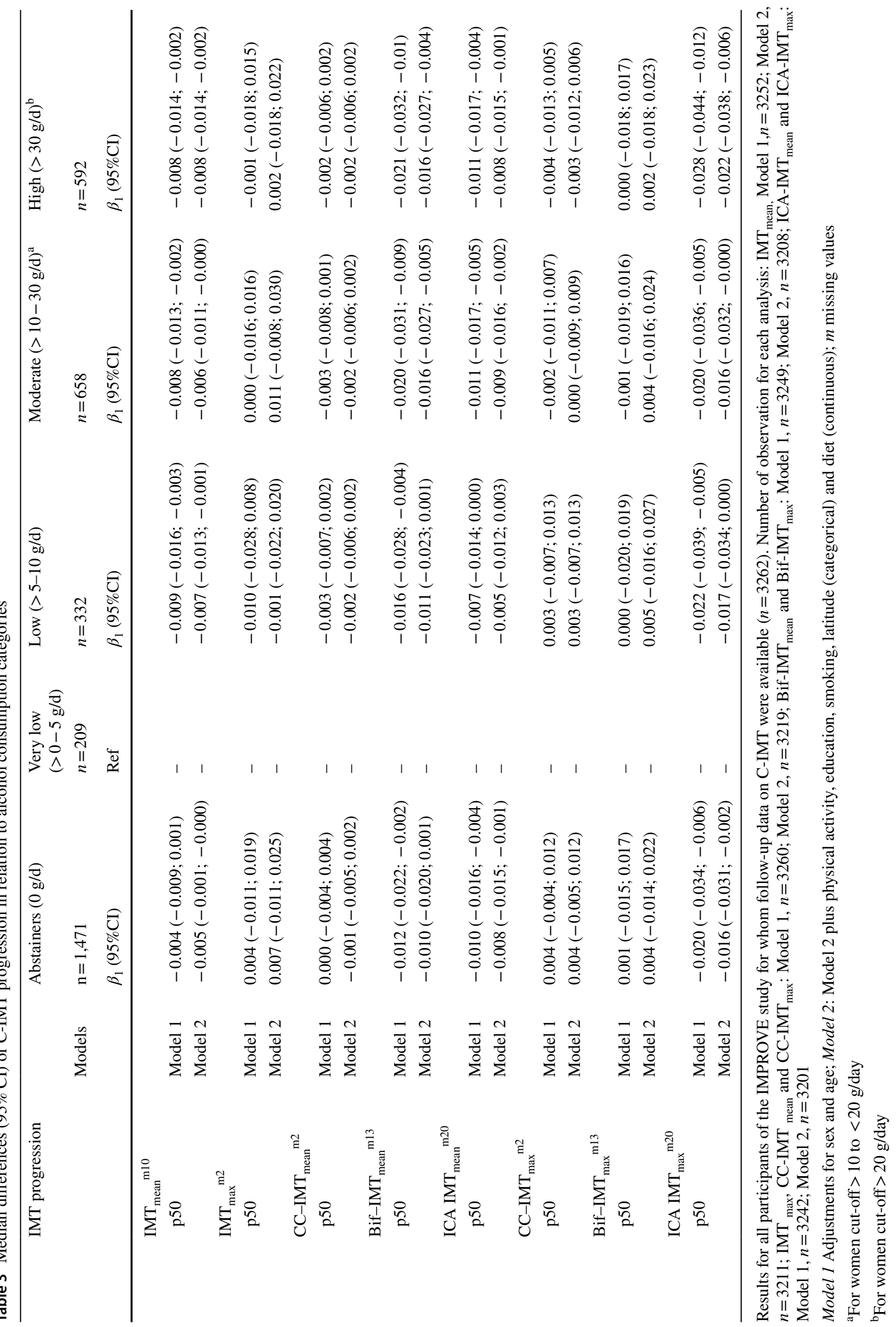


(A) Dose-response relationships between alcohol consumption and baseline C-IMT (p50)
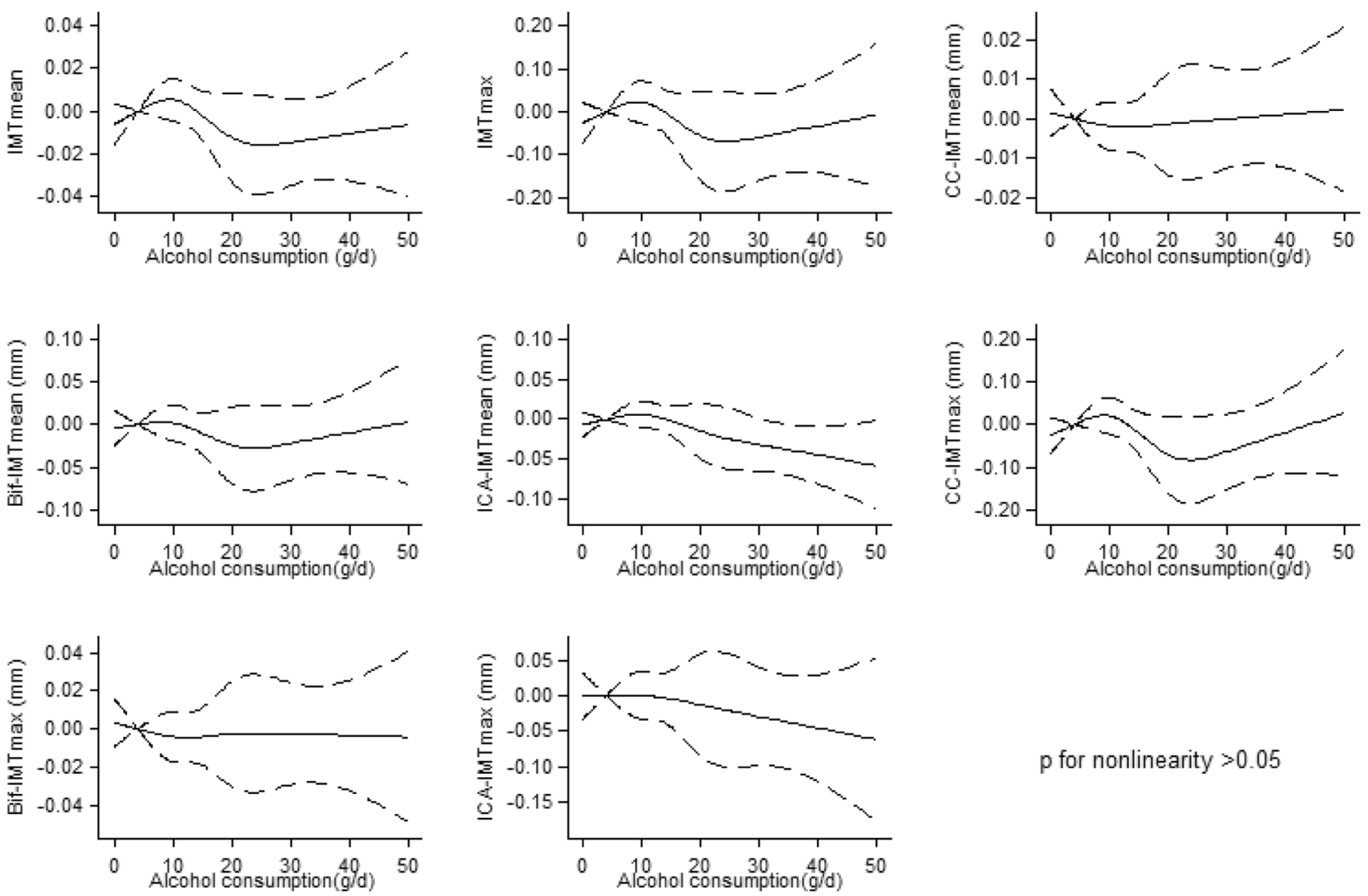

$p$ for nonlinearity $>0.05$

Fig. 1 a, b Dose-response relationships between alcohol consumption and each of the considered measurements of C-IMT (p50) at baseline (a) and progression (b). Solid lines: Restricted cubic splines adjusted for sex, age, physical activity, smoking, diet, and latitude, with knots located at fixed points of $\mathrm{g} / \mathrm{d}$ of alcohol consumption (4,

\section{Conclusion}

In this study population at high risk of CVD, moderate alcohol consumption was inversely associated with measurements of C-IMT and its progression. This finding supports
10, 20, 30). Dashed lines: $95 \%$ CI. 4 g/day was used as a reference point. $\mathrm{P}$ for nonlinearity was obtained testing the nullity of the coefficients associated with the second, third and fourth spline basis. For a better readability of the graphs, we excluded participants with alcohol consumption $>50 \mathrm{~g} / \mathrm{d}$

the hypothesis of a vascular protective effect exerted by moderate alcohol consumption. However, for clinical implications, it is important to consider that moderate alcohol consumption may increase risk of other diseases such as cancer. 
(B) Dose-response relationships between alcohol consumption and C-IMT progression (p50)
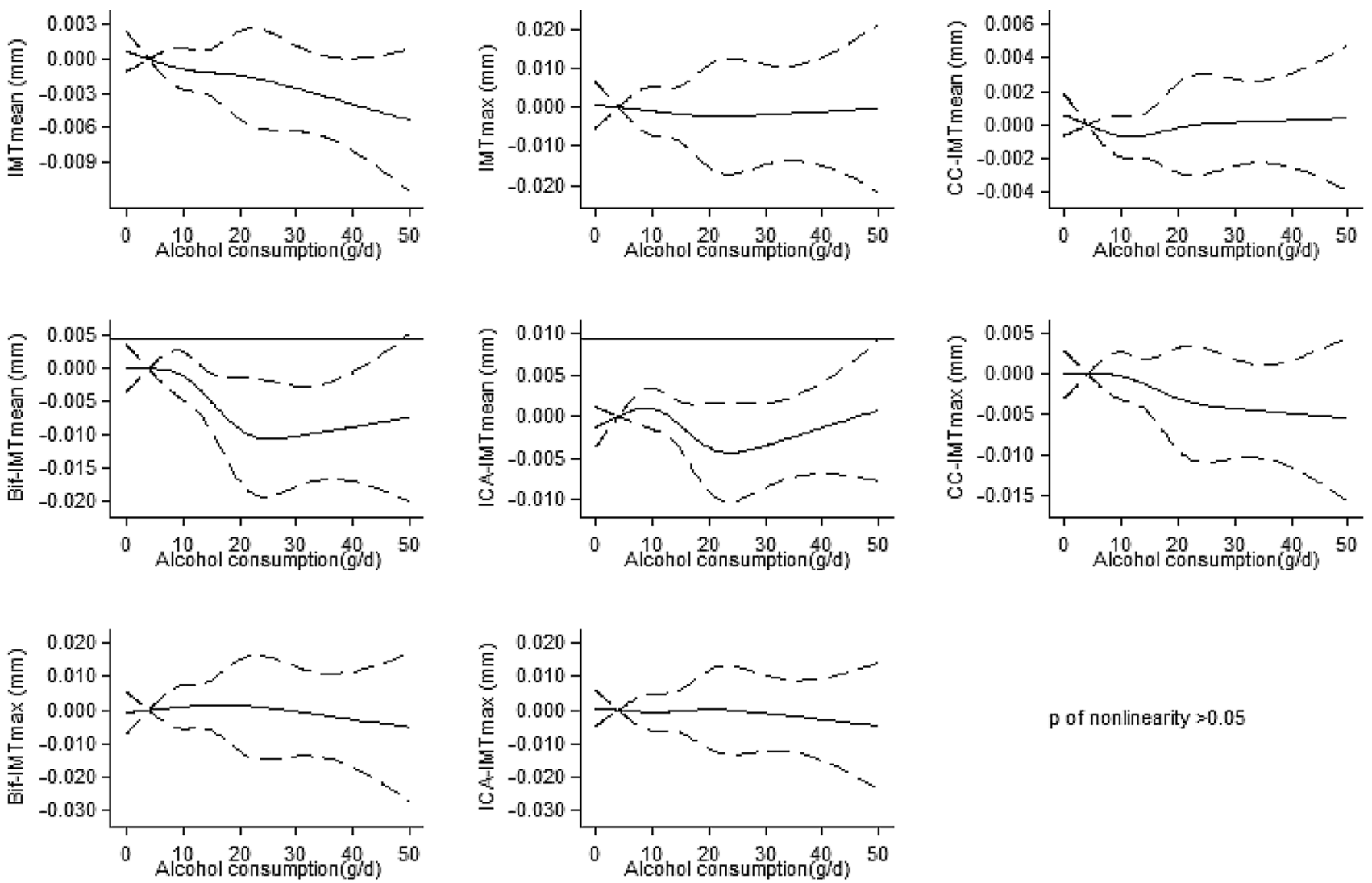

p of nonlinearity $>0.05$

Fig. 1 (continued)

Ackowledgements Open access funding provided by Karolinska Institute. The authors are deeply thankful to all the participants enrolled in the IMPROVE study. We also thank Gigante Bruna and Discacciati Andrea for their scientific support.

Author contribution All authors contributed substantively to this work. FL conceptualized the study and performed statistical analyses. FL and KL were involved in the interpretation of the results and drafted the report. All authors were involved in reviewing and editing of the manuscript and approved it.

Funding This study was supported by the European Commission (Contract number: QLG1-CT-2002-00896), the Swedish HeartLung Foundation, the Swedish Research Council (project 8691 and 0593), the Swedish Foundation for Strategic Research, the Stockholm County Council (project 562183), and the British Heart Foundation (RG2008/008). RJS is supported by a UKRI Innovation-HDR-UK Fellowship (MR/S003061/1).

\section{Compliance with ethical standards}

Conflict of interest The authors declare that there is no conflict of interest.
Open Access This article is licensed under a Creative Commons Attribution 4.0 International License, which permits use, sharing, adaptation, distribution and reproduction in any medium or format, as long as you give appropriate credit to the original author(s) and the source, provide a link to the Creative Commons licence, and indicate if changes were made. The images or other third party material in this article are included in the article's Creative Commons licence, unless indicated otherwise in a credit line to the material. If material is not included in the article's Creative Commons licence and your intended use is not permitted by statutory regulation or exceeds the permitted use, you will need to obtain permission directly from the copyright holder. To view a copy of this licence, visit http://creativecommons.org/licenses/by/4.0/.

\section{References}

1. Libby P, Ridker PM, Hansson GK (2011) Progress and challenges in translating the biology of atherosclerosis. Nature 473(7347):317-325

2. Pant $\mathrm{S}$ et al (2014) Inflammation and atherosclerosis-revisited. J Cardiovasc Pharmacol Ther 19(2):170-178

3. Nezu $T$ et al (2016) Carotid intima-media thickness for atherosclerosis. J Atheroscler Thromb 23(1):18-31 
4. Brien SE et al (2011) Effect of alcohol consumption on biological markers associated with risk of coronary heart disease: systematic review and meta-analysis of interventional studies. BMJ 342:d636

5. Huang $\mathrm{Y}$ et al (2017) Moderate alcohol consumption and atherosclerosis : Meta-analysis of effects on lipids and inflammation. Wien Klin Wochenschr 129(21-22):835-843

6. Chiva-Blanch G et al (2015) Effects of alcohol and polyphenols from beer on atherosclerotic biomarkers in high cardiovascular risk men: a randomized feeding trial. Nutr Metab Cardiovasc Dis 25(1):36-45

7. Holmes MV et al (2014) Association between alcohol and cardiovascular disease: mendelian randomisation analysis based on individual participant data. BMJ 349:g4164

8. Wurtz P et al (2016) Metabolic profiling of alcohol consumption in 9778 young adults. Int J Epidemiol 45(5):1493-1506

9. Bo P et al (2001) Effects of moderate and high doses of alcohol on carotid atherogenesis. Eur Neurol 45(2):97-103

10. Ferrieres J et al (1999) Carotid intima-media thickness and coronary heart disease risk factors in a low-risk population. J Hypertens 17(6):743-748

11. Fujisawa $\mathrm{M}$ et al (2008) Factors associated with carotid atherosclerosis in community-dwelling oldest elderly aged over 80 years. Geriatr Gerontol Int 8(1):12-18

12. Kiechl S et al (1998) Alcohol consumption and atherosclerosis: what is the relation? Prospective results from the Bruneck Study. Stroke 29(5):900-907

13. Kim MK et al (2014) Harmful and beneficial relationships between alcohol consumption and subclinical atherosclerosis. Nutr Metab Cardiovasc Dis 24(7):767-776

14. Schminke U et al (2005) Association between alcohol consumption and subclinical carotid atherosclerosis: the Study of Health in Pomerania. Stroke 36(8):1746-1752

15. Mukamal KJ et al (2003) Alcohol consumption and carotid atherosclerosis in older adults: the Cardiovascular Health Study. Arterioscler Thromb Vasc Biol 23(12):2252-2259

16. Lee $\mathrm{YH}$ et al (2009) Alcohol consumption and carotid artery structure in Korean adults aged 50 years and older. BMC Public Health 9:358

17. Xie X et al (2012) Alcohol consumption and carotid atherosclerosis in China: the Cardiovascular Risk Survey. Eur J Prev Cardiol 19(3):314-321

18. Jerrard-Dunne $P$ et al (2003) Interleukin-6 promoter polymorphism modulates the effects of heavy alcohol consumption on early carotid artery atherosclerosis: the Carotid Atherosclerosis Progression Study (CAPS). Stroke 34(2):402-407

19. Marques-Vidal $P$ et al (2006) Lack of association between ADH3 polymorphism, alcohol intake, risk factors and carotid intimamedia thickness. Atherosclerosis 184(2):397-403

20. Moon J et al (2018) Casual alcohol consumption is associated with less subclinical cardiovascular organ damage in Koreans: a cross-sectional study. BMC Public Health 18(1):1091

21. Knoflach $\mathrm{M}$ et al (2003) Cardiovascular risk factors and atherosclerosis in young males: ARMY study (Atherosclerosis RiskFactors in Male Youngsters). Circulation 108(9):1064-1069

22. Zyriax BC et al (2010) Association between alcohol consumption and carotid intima-media thickness in a healthy population: data of the STRATEGY study (Stress, Atherosclerosis and ECG Study). Eur J Clin Nutr 64(10):1199-1206

23. Kauhanen J et al (1999) Pattern of alcohol drinking and progression of atherosclerosis. Arterioscler Thromb Vasc Biol 19(12):3001-3006

24. Rantakomi SH et al (2009) Binge drinking and the progression of atherosclerosis in middle-aged men: an 11-year follow-up. Atherosclerosis 205(1):266-271

25. Juonala $\mathrm{M}$ et al (2009) Alcohol consumption is directly associated with carotid intima-media thickness in Finnish young adults: the Cardiovascular Risk in Young Finns Study. Atherosclerosis 204(2):e93-e98

26. Kesse-Guyot E et al (2010) Associations between dietary patterns and arterial stiffness, carotid artery intima-media thickness and atherosclerosis. Eur J Cardiovasc Prev Rehabil 17(6):718-724

27. Fujii K et al (2003) Risk factors for the progression of early carotid atherosclerosis in a male working population. Hypertens Res 26(6):465-471

28. Markus RA et al (1997) Influence of lifestyle modification on atherosclerotic progression determined by ultrasonographic change in the common carotid intima-media thickness. Am J Clin Nutr 65(4):1000-1004

29. McClelland RL et al (2008) Alcohol and coronary artery calcium prevalence, incidence, and progression: results from the Multi-Ethnic Study of Atherosclerosis (MESA). Am J Clin Nutr 88(6):1593-1601

30. Mowbray PI et al (1997) Cardiovascular risk factors for early carotid atherosclerosis in the general population: the Edinburgh Artery Study. J Cardiovasc Risk 4(5-6):357-362

31. Spring B et al (2014) Healthy lifestyle change and subclinical atherosclerosis in young adults: Coronary Artery Risk Development in Young Adults (CARDIA) study. Circulation 130(1):10-17

32. Britton AR et al (2017) Alcohol consumption and common carotid intima-media thickness: the USE-IMT Study. Alcohol Alcohol 52(4):483-486

33. Zureik M et al (2004) Alcohol consumption and carotid artery structure in older French adults: the Three-City Study. Stroke 35(12):2770-2775

34. Demirovic J et al (1993) Alcohol consumption and ultrasonographically assessed carotid artery wall thickness and distensibility. The Atherosclerosis Risk in Communities (ARIC) Study Investigators. Circulation 88(6):2787-2793

35. Djousse L et al (2002) Influence of apolipoprotein E, smoking, and alcohol intake on carotid atherosclerosis: National Heart, Lung, and Blood Institute Family Heart Study. Stroke 33(5):1357-1361

36. Kelso-Chichetto NE et al (2017) The impact of long-term moderate and heavy alcohol consumption on incident atherosclerosis among persons living with HIV. Drug Alcohol Depend 181:235-241

37. Baldassarre D et al (2010) Cross-sectional analysis of baseline data to identify the major determinants of carotid intima-media thickness in a European population: the IMPROVE study. Eur Heart J 31(5):614-622

38. Baldassarre D et al (2013) Progression of carotid intimamedia thickness as predictor of vascular events: results from the IMPROVE study. Arterioscler Thromb Vasc Biol 33(9):2273-2279

39. von Elm E et al (2007) The Strengthening the Reporting of Observational Studies in Epidemiology (STROBE) statement: guidelines for reporting observational studies. Lancet 370(9596): 1453-1457

40. Turner C (1990) How much alcohol is in a 'standard drink'? An analysis of 125 studies. Br J Addict 85(9):1171-1175

41. Beyerlein A (2014) Quantile regression-opportunities and challenges from a user's perspective. Am J Epidemiol 180(3):330-331

42. ESC (2015) Simova Intima-media thickness: Appropriate evaluation and proper measurement, described. E J ESC Counc Cardiol Pract 13:21

43. Spence JD, Pilote L (2015) Importance of sex and gender in atherosclerosis and cardiovascular disease. Atherosclerosis 241(1):208-210

44. Erol A, Karpyak VM (2015) Sex and gender-related differences in alcohol use and its consequences: Contemporary knowledge and future research considerations. Drug Alcohol Depend 156:1-13 
45. Calderon RM et al (2010) Statins in the treatment of dyslipidemia in the presence of elevated liver aminotransferase levels: a therapeutic dilemma. Mayo Clin Proc 85(4):349-356

46. Toda $\mathrm{M}$ et al (2017) Low dose of alcohol attenuates pro-atherosclerotic activity of thrombin. Atherosclerosis 265:215-224

47. Vasdev S, Gill V, Singal PK (2006) Beneficial effect of low ethanol intake on the cardiovascular system: possible biochemical mechanisms. Vasc Health Risk Manag 2(3):263-276

48. Mackinnon AD et al (2004) Rates and determinants of site-specific progression of carotid artery intima-media thickness: the carotid atherosclerosis progression study. Stroke 35(9):2150-2154
49. Stockwell T, Chikritzhs T (2013) Commentary: another serious challenge to the hypothesis that moderate drinking is good for health? Int J Epidemiol 42(6):1792-1794

50. Naimi TS et al (2017) Selection biases in observational studies affect associations between 'moderate' alcohol consumption and mortality. Addiction 112(2):207-214

51. Liu W et al (2011) Differential effects of daily-moderate versus weekend-binge alcohol consumption on atherosclerotic plaque development in mice. Atherosclerosis 219(2):448-454

\section{Affiliations}

\section{Federica Laguzzi ${ }^{1}$. Damiano Baldassarre ${ }^{2,3} \cdot$ Fabrizio Veglia $^{3} \cdot$ Rona J. Strawbridge $^{4,5} \cdot$ Steve E. Humphries $^{6}$. Rainer Rauramaa ${ }^{7,8} \cdot$ Andries J. Smit $^{9} \cdot$ Philippe Giral $^{10}$. Angela Silveira ${ }^{5}$. Elena Tremoli ${ }^{3}$. Anders Hamsten ${ }^{5}$. Ulf de Faire ${ }^{1,11} \cdot$ Paolo Frumento $^{12} \cdot$ Karin Leander $^{1}$ on behalf of IMPROVE Study group}

1 Unit of Cardiovascular and Nutritional Epidemiology, Institute of Environmental Medicine, Karolinska Institutet, Nobels väg 13, Box 210, 17177 Stockholm, Sweden

2 Department of Medical Biotechnology and Translational Medicine, Università degli Studi di Milano, Milan, Italy

3 Centro Cardiologico Monzino, IRCCS, Milan, Italy

4 Institute of Mental Health and Wellbeing, Mental Health and Wellbeing, University of Glasgow, Glasgow, UK

5 Cardiovascular Medicine Unit, Department of Medicine, Karolinska Institutet, Stockholm, Sweden

6 Centre for Cardiovascular Genetics, Institute Cardiovascular Science, University College London, London, UK

7 Foundation for Research in Health Exercise and Nutrition, Kuopio Research Institute of Exercise Medicine, Kuopio, Finland
8 Department of Clinical Physiology and Nuclear Medicine, Kuopio University Hospital, Kuopio, Finland

9 Department of Medicine, University Medical Centre Groningen, University of Groningen, Groningen, The Netherlands

10 Assistance Publique-Hôpitaux de Paris, Service Endocrinologie-Métabolisme, Groupe Hospitalier Pitié-Salpétrière, Unités de Prévention Cardiovasculaire, Paris, France

11 Department of Cardiology, Karolinska University Hospital, Stockholm, Sweden

12 Unit of Biostatistics, Institute of Environmental Medicine, Karolinska Institutet, Nobels väg 13, 17177 Stockholm, Sweden 